\section{ORIGINAL RESEARCH}

\author{
J.J.S. Shankar \\ C. Lum
}

N. Parikh

M. dos Santos

\title{
Long-Term Prospective Follow-Up of Intracranial Aneurysms Treated with Endovascular Coiling Using Contrast-Enhanced MR Angiography
}

BACKGROUND AND PURPOSE: Catheter angiography has been the criterion standard for follow-up evaluation of coiled intracranial aneurysms. In our center, CE-MRA has been used to evaluate aneurysm recanalization. Our aim was to investigate the feasibility and usefulness of a CE-MRA protocol for following patients with intracranial aneurysms treated with endovascular coiling

MATERIALS AND METHODS: From September 2003 to December 2006, 134 aneurysms were treated by endovascular coiling in 124 patients by using detachable coils. These patients were followed with CE-MRA at 3 months, 15 months, and 3 and 5 years. MRAs were analyzed by 2 interventional neuroradiologists. Findings were assigned to 3 categories: complete obliteration (class 1), residual neck (class 2), and residual aneurysm (class 3$)$.

RESULTS: Initially, CE-MRA demonstrated 67 (50\%) complete obliterations (class 1), 57 (41.79\%) residual necks (class 2), and 8 (5.97\%) residual aneurysms (class 3$)$. No patient experienced rebleed during the follow-up period. A total of 214 patient-years of follow-up were obtained (range, 0-53 months). Two (1.49\%) patients died after the follow-up, and 11 (8.21\%) patients were lost to follow-up. On follow-up, $76(56.72 \%)$ patients showed stable results. Fifty-six $(41.79 \%)$ aneurysms showed change in their obliteration pattern. Of these 56, 47 demonstrated recanalization and $9(6.72 \%)$ showed further obliteration. Most of the aneurysms that showed change in their obliteration remained stable on follow-up. Only 11 (8.21\% of the total and $23.4 \%$ of those who showed recanalization) patients underwent recoiling or clipping.

CONCLUSIONS: CE-MRA can be used in routine practice to follow-up aneurysm recanalization noninvasively. CE-MRA permits close-interval follow-up and may show more filling of the aneurysm neck or sac than DSA.

ABBREVIATIONS: $\mathrm{ACA}=$ anterior cerebral artery; $\mathrm{AcomA}=$ anterior communicating artery; $\mathrm{AICA}=$ anterior inferior cerebellar artery; CE-MRA = contrast-enhanced MRA; CTA $=$ CT angiography; DSA = digital subtraction angiography; FLASH = fast low-angle shot; ICA = internal carotid artery; ISAT = International Subarachnoid Aneurysm Trial; MCA = middle cerebral artery; MIP = maximum intensity projection; MRA = MR angiography; PCA = posterior cerebral artery; PcomA = posterior communicating artery; PICA = posterior inferior cerebellar artery; $\mathrm{SAH}=$ subarachnoid hemorrhage; TOF $=$ time of flight

E ndovascular treatment has now become a major option in the treatment of the intracranial aneurysms. ${ }^{1}$ One of the criticisms of endovascular aneurysm coiling is the recurrence rate compared with surgical clipping. ${ }^{2-6}$

Because many aneurysms are being treated by endovascular coiling, long-term follow-up with a reliable noninvasive imaging technique is desirable. DSA has traditionally been the technique of choice for follow-up of treated aneurysms. However, DSA is invasive and still carries with it a measurable degree of morbidity. ${ }^{7}$ Other issues to consider with DSA are patient discomfort, cost, and radiation exposure.

MR angiography has been performed by several groups to

Received October 18, 2009; accepted after revision December 28.

From the Department of Diagnostic Imaging, The Ottawa Hospital, Ottawa, Ontario, Canada.

Paper previously presented in part at: Annual Meeting of the American Society of Neuroradiology, June 9-14, 2007; Chicago, Illinois; C. Lum and N. Parikh were recipients of the 2006 Norman Leeds Award, from the Eastern Neuroradiological Society for "Contrast-Enhanced MRA for the Follow-Up of Coiled Aneurysms."

Please address correspondence to Cheemun Lum, MD, Department of Diagnostic Imaging, The Ottawa Hospital, Ottowa, Ontario, K1Y 4E9, Canada; e-mail: chlum@ ottawahospital.on.ca

DOI 10.3174/ajnr.A2064

monitor coiled aneurysms. ${ }^{8-16}$ In a few reports, CE-MRA has been found to be better compared with TOF-MRA. ${ }^{8,9,14,15}$ CE-MRA has been compared with DSA and has been found to have a very good correlation with it in the same subset of patients. ${ }^{8,11,13,14,16}$ Because of the growing body of literature supporting CE-MRA for following coiled aneurysms, our center developed a policy of using CE-MRA as the primary imaging technique to detect recurrences. There is still debate regarding the best method to follow coiled aneurysms. The purpose of our study was to assess our long-term practical experience by using CE-MRA to evaluate the baseline postcoiling appearance of aneurysms and to monitor recurrences.

\section{Materials and Methods}

This study was reviewed and approved by our institutional review board. All cases of intracranial aneurysms coiled between September 2003 and December 2006 were prospectively followed with CE-MRA at intervals of 0,3 , and 15 months and 3 and 5 years. Shorter intervals of CE-MRA were performed if there was clinical concern.

\section{Endovascular Treatment}

Endovascular embolization of the aneurysms was performed by using detachable coils on a DSA system (Axiom Artis; Siemens, Erlangen, 
Germany). All patients were treated under general anesthesia. A selection of coils, balloons, and stents was used according to the availability and preferences of the surgeons. The immediate postcoiling DSA images served as a reference for follow-up imaging. The initial results of endovascular coiling based on DSA were classified into 3 categories: complete obliteration (class 1), residual neck (class 2), and residual aneurysm (class 3$){ }^{17}$

\section{Follow-Up}

CE-MRA. All CE-MRAs were performed on a superconductiong 1.5T MR imaging system (Symphony V25 software, Siemens) with a standard head coil. A CE-MRA FLASH sequence was acquired with $k$-space sampling in 3D centric reordering $\left(6 / 8\right.$, flip angle $=35^{\circ}$ for gabdutrol [Gadovist; Bayer Schering Pharma, Berlin, Germany] and $25^{\circ}$ for gadodiamide [Omniscan; Nycomed, Amersham, Oslo, Norway], FOV of 23 , and matrix size of $448 \times 358$ ) in the coronal plane. The acquisition time was 1 minute 6 seconds. The acquisition volume was placed on the sagittal scout image so that the volume included the circle of Willis. The anteroposterior diameter was $61 \mathrm{~mm}$, and the section thickness was $0.7 \mathrm{~mm}$ with 96 partitions. A bolus of gadolinium chelate $(0.5 \mathrm{mmol} / \mathrm{kg}$ of body weight of Omniscan or $1 \mathrm{mmol} / \mathrm{kg}$ of body weight of Gadovist) was injected at a rate of $2 \mathrm{~mL} / \mathrm{s}$ by using an MR imaging-compatible power injector. The circulation time of contrast media from the antecubital vein to the arch of the aorta was estimated by using a Combined Application Reduction Exposure bolus (Siemens, Malvern, Pennsylvania) (2D turboFLASH sequence) before manually triggering the CE-MRA acquisition. Source images were then reconstructed by using an MIP algorithm. Multiple projections by using a large FOV were obtained every 15 degrees over 180 degrees in lateral and anteroposterior views.

The first CE-MRA was performed within a week of aneurysm coiling. The follow-up CE-MRAs were performed at 3 months, 1 year, 3 years, and 5 years. In cases in which the first CE-MRA suggested concern for aneurysm recanalization, we shortened the interval to the subsequent CE-MRA.

Image Analysis. All coiling procedures were performed by fellowship-trained neurointerventionists. The DSA images obtained at the end of the coiling procedure and CE-MRA were evaluated by interventional neuroradiologists who also perform endovascular coiling. The CE-MRA images provided to them were axial source images of the brain and MIP reconstructions of the appropriate artery associated with the coiled aneurysm. The DSA images provided were anteroposterior, lateral, and rotational projections obtained at the end of coiling. Findings of the residual neck or body were assigned to 3 categories: complete obliteration (class 1), residual neck (class 2), and residual aneurysm (class 3) according to the Roy and Milot classification. $^{17}$

Statistical Analysis. Different parameters regarding the location of the aneurysms and outcomes of the aneurysm treatment were taken into account in calculating the various percentages.

\section{Results}

\section{Patients}

A total of 124 consecutive patients ( 30 men, 94 women; mean age, 55.46 years; range, $22-82$ years) with 134 aneurysms were treated from 2003 to 2006 by endovascular coiling with detachable coils and were followed by using CE-MRA as shown in Table 1. Of these, $80(64.5 \%)$ patients had SAH. The aneu-

\begin{tabular}{lc}
\hline Table 1: Demographic data & No. (\%) \\
\hline & 124 (30 men, 94 women; mean age, \\
Patients & 55.4 years; range, 22-82 years) \\
& 134 \\
Aneurysms & $80(59.7)$ \\
SAH & $54(40.3)$ \\
No SAH & $97(72.4)$ \\
Anterior circulation & $25(18.7)$ \\
AcomA & $26(19.4)$ \\
ICA & $11(8.2)$ \\
ACA & $14(10.5)$ \\
MCA & $21(15.7)$ \\
PcomA & $37(27.6)$ \\
Posterior circulation & $28(20.9)$ \\
Basilar tip & $1(0.8)$ \\
AICA & $4(2.9)$ \\
PICA & $1(0.8)$ \\
PCA & $3(2.2)$ \\
Vertebral artery & $18(13.4)$ \\
Stents (Neuroform) & $2(1.6)$ \\
Death & $11(8.8)$ \\
Lost to follow-up & $111(89.5)$ \\
Follow-up &
\end{tabular}

\section{Table 2: CE-MRA: initial and follow-up results}

\begin{tabular}{lc}
\hline & No. $(\%)$ \\
\hline Initial obliteration & \\
Class 1 & $67(50)$ \\
Class 2 & $57(42.5)$ \\
Class 3 & $8(5.9)$ \\
Death & $2(1.6)$ \\
Lost to follow-up & $11(8.8)$ \\
Follow-up & $111(89.5)$ \\
Stable & $76(56.7)$ \\
Class 1 & $41(30.6)$ \\
Class 2 & $26(19.4)$ \\
Class 3 & $9(6.7)$ \\
Unstable & $56(41.8)$ \\
Recanalization & $47(35.1)$ \\
Classes 1-2 & $20(14.9)$ \\
Classes 1-3 & $6(4.5)$ \\
Classes 2-3 & $21(15.7)$ \\
Further obliteration & $9(6.7)$ \\
Classes 2-1 & $8(5.9)$ \\
Classes 3-2 & 0 \\
Classes 3-1 & $1(0.8)$ \\
Recanalization to class 3 after coiling & $27(20.2)$ \\
$<6$ Months & $16(11.9)$ \\
6-12 Months & $3(2.3)$ \\
$>12$ Months & $8(5.9)$ \\
Retreatment & $11(8.2)$ \\
\hline
\end{tabular}

rysms were located on the anterior circulation in 97 patients $(\mathrm{AcomA}=25, \mathrm{ICA}=26, \mathrm{ACA}=11, \mathrm{MCA}=14, \mathrm{PcomA}=$ 21 ) and in the posterior circulation in 37 patients (basilar artery $=28, \mathrm{AICA}=1, \mathrm{PICA}=4, \mathrm{PCA}=1$, vertebral artery $=$ $3)$. The aneurysms ranged from 2 to $25 \mathrm{~mm}$. All the aneurysms were coiled by using platinum coils. Eighteen aneurysms were coiled with stent assistance (Neuroform stents; Boston Scientific, Natick, Massachusetts).

\section{Initial Results}

Initially, CE-MRA demonstrated complete obliteration (class 1 ) in $67(50 \%)$, residual neck (class 2$)$ in $57(42.5 \%)$, and 

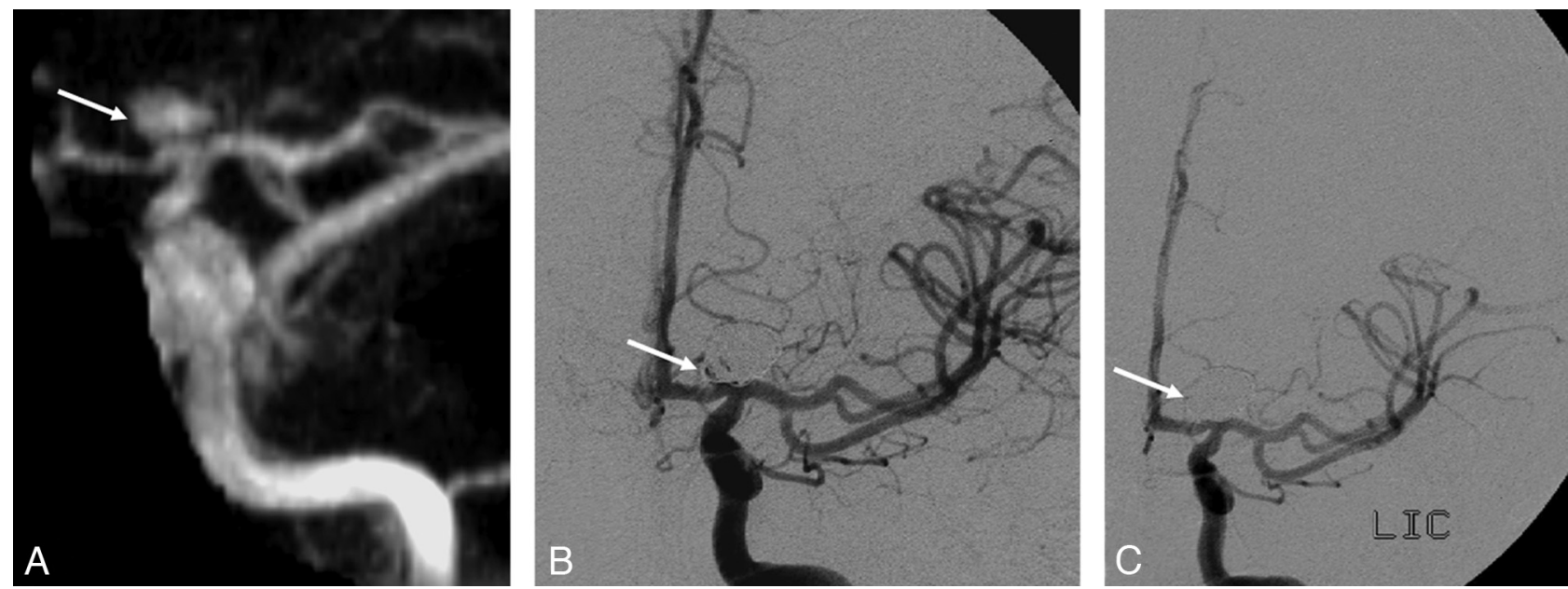

Fig 1. A, CE-MRA shows large recurrent class 3 aneurysm (arrow). $B$, Anteroposterior angiogram of the left ICA injection shows only minimal filling of the coiled aneurysm (arrow). $C$, Anteroposterior angiogram of the left ICA injection after recoiling (arrow) by using two $5 \mathrm{~mm} \times 20 \mathrm{~cm}$ and 1 each of $4 \mathrm{~mm} \times 10 \mathrm{~cm}, 3 \mathrm{~mm} \times 8 \mathrm{~cm}$, and $2 \mathrm{~mm} \times 6 \mathrm{~cm}$ coils. This shows the helmet effect of coils, which obscures the filling of the coiled aneurysm on the conventional angiogram.

residual aneurysms (class 3 ) in 8 (5.9\%) aneurysms. The other $2(1.6 \%)$ patients died (Table 2 ).

\section{Follow-Up}

A total of 214 patient-years of follow-up (Table 2) were obtained (range, $0-53$ months). None of the patients in our group experienced rebleed during the follow-up period. Two (1.49\%) patients died on follow-up, and 11 (8.21\%) patients were lost to follow-up (91.13\% followed). Two patients had suboptimal CE-MRA because of associated clipping and stent placement. In the other 18 patients treated with the assistance of Neuroform stents, the quality of CE-MRA was judged to be satisfactory to good. Two patients could not undergo MR imaging due to claustrophobia and a pacemaker.

On follow-up, $56.72 \%$ (76 aneurysms) showed stable results. Of these, $30.6 \%$ (41 aneurysms) demonstrated stable class $1,19.4 \%$ (26 aneurysms) had stable class 2 , and $6.72 \%$ (9 aneurysms) had stable class 3 obliteration.

A total of $41.79 \%$ (56 aneurysms) demonstrated a change in their obliteration pattern. Of these, $83.93 \%$ (47 aneurysms) demonstrated recanalization and $6.72 \%$ ( 9 aneurysms) showed further obliteration. Of the 47 recanalized aneurysms, $20(14.93 \%)$ changed from class 1 to class 2 ; six $(4.48 \%)$, from class 1 to class 3 ; and 21 (15.67\%), from class 2 to class 3 . Of the 9 aneurysms that underwent further obliteration, 8 (5.97\%) changed from class 2 to class 1 , and $1(0.75 \%)$ changed from class 3 to class 1 . Most of the aneurysms that demonstrated a change in their obliteration remained stable on follow-up. A total of $11(8.21 \%$ of the total and $23.4 \%$ of those that showed recanalization) patients underwent recoiling or clipping.

Of the 27 aneurysms that showed significant recanalization to class 3,59\% $(n=16)$ were recanalized within 6 months of coiling, $70 \%(n=19)$ were recanalized within 12 months, and approximately $30 \%(n=8)$ were recanalized after 12 months of follow-up. The latest recanalization observed was after 29 months of follow-up. However, most aneurysms that remained stable after 1 year of follow-up were most likely to remain so afterward.

\section{Discussion}

In the post-ISAT ${ }^{1}$ era, many aneurysms are treated by endovascular coiling procedures. This practice raises the question of the durability of results and how to best evaluate patients in the long term. With endovascular coiling, a residual neck is not an uncommon finding. The controversy lies in the stability and the natural history of these so-called partially coiled aneurysms with residual necks. ${ }^{2,17}$ An imaging technique that is noninvasive and can be performed at shorter intervals depending on the degree of recanalization is desirable to follow these patients closely. Various studies have demonstrated the value of CE-MRA in comparison with DSA for the evaluation of coiled aneurysms on follow-up. ${ }^{8,11,13,14,16}$ It was not our intent to compare DSA and CE-MRA because this comparison has been extensively investigated. Rather, our center adopted a noninvasive paradigm to monitor coiled aneurysms with CEMRA a number of years ago. Because controversy still exists regarding whether DSA or CE-MRA is more appropriate, we believe that it is important to share our long-term results using CE-MRA as our preferred surveillance method.

In our study, approximately $40 \%$ of our aneurysms demonstrated a change in obliteration pattern. Most of these, 35\%, had further recanalization, which is similar to the results of other reported series in the literature. ${ }^{18}$ In our experience, because CE-MRA is noninvasive, it lends itself to more frequent interval monitoring of these patients with significant early recanalizations. More data points, conceptually, lead to improved understanding of the rate of recanalization.

Diagnostic angiography still caries a complication rate of approximately $1 \% .{ }^{7}$ In our center, CE-MRA has completely replaced DSA for follow-up of the coiled aneurysms. CE-MRA may also show more filling compared with DSA. ${ }^{16}$ We have seen cases in which DSA appears to show a class 1 obliteration; however, CE-MRA demonstrates class 2 filling. This may be due to the inherent accuracy of CE-MRA compared with DSA or, less likely, may reflect a change in local aneurysmal environment occurring between the 2 studies. In a small number of cases, CE-MRA may demonstrate more filling of an aneurysm than DSA owing to the "helmet-type" effect (Fig 1). ${ }^{16}$ In 

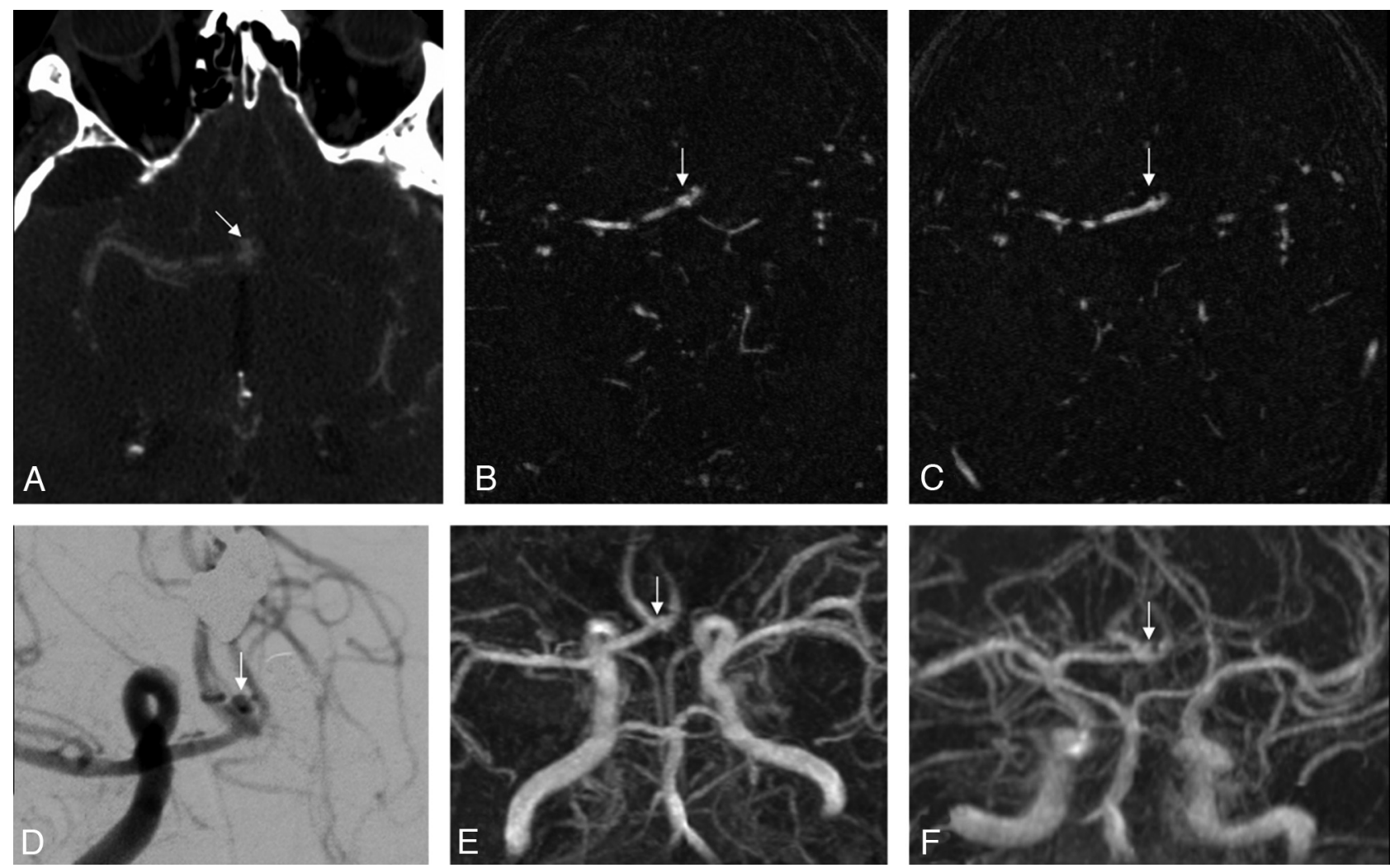

Fig 2. $A$ and $D$, Axial base image of the CT angiography $(A)$ performed at presentation of the patient shows an AcomA aneurysm, which is further confirmed on the subsequent conventional angiogram $(D)$. $B$ and $E$, The base image $(B)$ and the MIP image $(E)$ on CE-MRA performed 3 months after coiling show no evidence of residual filling. $C$ and $F$, The base image $(C)$ and the MIP image $(F)$ on CE-MRA performed at 20 months' follow-up show a small amount of residual filling of the aneurysm near the neck, which can be more confidently seen compared with the base image of the initial CT angiogram $(A)$.

our experience with retreatment of these helmet-type remnants, the size and number of coils that can be deployed in the remnant more accurately reflect the findings at CE-MRA compared with DSA (Fig 1). This is likely related to the inability of $\mathrm{X}$-rays to penetrate the platinum shell of coils.

Several authors have compared MRA and DSA ${ }^{8,11,13,14,16}$ and also different techniques (3D-TOF and CE-MRA) of MRA. ${ }^{8,9,14,15}$ We report the follow-up using only CE-MRA to evaluate coiled aneurysms. Our initial complete (class 1 ) obliteration rate $(50 \%)$ is similar to that in the literature. ${ }^{9,11,14,15,17}$ We emphasize the point that our degree of initial obliteration is at the predischarge CE-MRA. Our experience has led us to conclude that CE-MRA assesses neck remnants more accurately compared with DSA. This may explain a slight difference in our obliteration rates compared with those in other series. ${ }^{9,11,14,15,17}$ Approximately 57\% of our patients had stable results. Most of the $42 \%$ of patients who demonstrated changes in the obliteration pattern remained stable with time. Twenty percent of our patients progressed to class 3 recanalization following coiling. However, most of these patients required no treatment because their class 3 status remained stable. Eight percent of our patients required retreatment, a figure similar to that in other published rates. ${ }^{2,17}$ We found that most recanalization to class 3 occurred $<6$ months from treatment, similar to that in the current literature. ${ }^{4}$ However, aneurysms of 8 of 27 (29.63\%) patients recanalized to class 3 after 12 months. This finding emphasizes the importance of long-term follow-up as described in other studies. ${ }^{11,18}$

In our center, all patients with suspected aneurysmal SAH undergo CTA for preoperative evaluation. In many cases, treatment decisions regarding coiling or clipping are feasible. This allows coordinated planning of anesthesia without an interposed DSA. ${ }^{19}$ The source images from CTA allow delineation of the aneurysm in an axial plain, the identical plane in which our CE-MRA source images are acquired. In addition, our coiling follow-up CE-MRA studies are interpreted solely by interventional neuroradiologists, who also perform aneurysm coiling. This continuity of imaging pre- and postcoiling is important (Fig 2). It allows the evaluators an understanding of the aneurysm location in relation to the parent artery and adjacent arteries in the axial plane. The treating physicians are often the same ones performing the CE-MRA interpretation and thus have an understanding of the portion of the aneurysm in which it was difficult to achieve optimal packing. This paradigm allows excellent correlation between the axial CTA pretreatments scans, the DSA at coiling, and axial CE-MRA source images at follow-up.

In the past, there has been significant focus on aneurysm recanalization. ${ }^{2}$ In our study, $8 \%$ of coiled aneurysms were retreated. Our study is similar to other series in confirming that most aneurysm remnants do not require retreatment and can be followed. ${ }^{17}$ Most of the aneurysms in our series that were stable after 1 year of follow-up continued to remain stable. CE-MRA is an ideal method to track patients noninvasively long term to confirm stability. With CE-MRA as the sole technique to follow coiled aneurysms, there were no bleeds or rebleeds in the study period.

Stent-assisted coiling has evolved to become an important 
tool in aneurysm coiling. In our series, CE-MRA was satisfactory to good in evaluating aneurysms coiled with Neuroform stent assistance. There tends to be a small amount of magnetic susceptibility artifacts at the stent margins, which does not tend to affect imaging interpretation. ${ }^{20}$

Despite these advantages, CE-MRA carries a very low risk of nephrogenic systemic fibrosis. ${ }^{2,21-22}$ This complication can be avoided by using judgment in administering contrast agent in patients with chronic renal diseases. In these cases, TOFMRA may be a reasonable option.

Our study is limited by its observational nature, the lack of a control group, and a relatively small number of patients. The intention, however, was not to compare CE-MRA with DSA but to evaluate the practical utility of CE-MRA in following coiled aneurysms.

\section{Conclusions}

CE-MRA is a practical noninvasive method to assess and monitor aneurysm recanalization. CE-MRA permits close interval follow-up and may show more filling of the aneurysm neck or sac than DSA. The noninvasive benefits of CE-MRA allow long-term follow-up at very low risk.

\section{References}

1. Molyneux A, Kerr R, Stratton I, for the International Subarachnoid Aneurysm Trial (ISAT) Collaborative Group. International Subarachnoid Aneurysm Trial (ISAT) of neurosurgical clipping versus endovascular coiling in 2143 patients with ruptured intracranial aneurysms: a randomised trial. Lancet 2002;360:1267-74

2. Molyneux AJ, Kerr RS, Yu LM, et al, for the International Subarachnoid Aneurysm Trial (ISAT) Collaborative Group. International subarachnoid aneurysm trial (ISAT) of neurosurgical clipping versus endovascular coiling in 2143 patients with ruptured intracranial aneurysms: a randomised comparison of effects on survival, dependency, seizures, rebleeding, subgroups and aneurysm occlusion. Lancet 2005;366:809-17

3. Feuerberg I, Lindquist C, Lindqvist M, et al. Natural history of postoperative aneurysm rests. J Neurosurg 1987;66:30-34

4. Thornton J, Debrun GM, Aletich VA, et al. Follow-up angiography of intracranial aneurysms treated with endovascular placement of Guglielmi detachable coils. Neurosurgery 2002;50:239-49

5. Asgari S, Wanke I, Schoch B, et al. Recurrent hemorrhage after initially complete occlusion of intracranial aneurysms. Neurosurg Rev 2003;26:269-74

6. Hope JK, Byrne JV, Molyneux AJ. Factors influencing successful angiographic occlusion of aneurysms treated by coil embolization. AJNR Am J Neuroradiol 1999;20:391-99
7. Willinsky RA, Taylor SM, terBrugge K, et al. Neurologic complications of cerebral angiography: prospective analysis of 2,899 procedures and review of the literature. Radiology 2003;227:522-28

8. Leclerc X, Navez J, Gauvrit J, et al. Aneurysms of the anterior communicating artery treated with Guglielmi detachable coils: follow-up with contrast-enhanced MR angiography. AJNR Am J Neuroradiol 2002;23:1121-27

9. Cottier J, Bleuzen-Couthon A, Gallas S, et al. Intracranial aneurysms treated with Guglielmi detachable coils: is contrast material necessary in the follow-up with 3D time-of-flight MR angiography? AJNR Am J Neuroradiol 2003;24:1797-803

10. Yamada N, Hayashi K, Murao K, et al. Time-of-Flight MR angiography targeted to coiled intracranial aneurysms is more sensitive to residual flow than is digital subtraction angiography. AJNR Am J Neuroradiol 2004;25:1154-57

11. Gauvrit J, Leclerc X, Pernodet $M$, et al. Intracranial aneurysms treated with Guglielmi detachable coils: usefulness of 6-month imaging follow-up with contrast-enhanced MR angiography. AJNR Am J Neuroradiol 2005;26:515-21

12. Majoie CB, Sprengers ME, van Rooij WJ, et al. MR angiography at $3 T$ versus digital subtraction angiography in the follow-up of intracranial aneurysms treated with detachable coils. AJNR Am J Neuroradiol 2005;26:1349-56

13. Gauvrit J, Leclerc $X$, Caron $S$, et al. Intracranial aneurysms treated with Guglielmi detachable coils: imaging follow-up with contrast-enhanced MR angiography. Stroke 2006;37:1033-37

14. Pierot L, Delcourt C, Bouquigny F, et al. Follow-up of intracranial aneurysms selectively treated with coils: prospective evaluation of contrast-enhanced MR angiography. AJNR Am J Neuroradiol 2006;27:744-49

15. Anzalone N, Scomazzoni F, Cirillo M, et al. Follow-up of coiled cerebral aneurysms at 3T: comparison of 3D time-of-flight MR angiography and contrastenhanced MR angiography. AJNR Am J Neuroradiol 2008;29:1530-36

16. Agid R, Willinsky RA, Lee SK, et al. Characterization of aneurysm remnants after endovascular treatment: contrast-enhanced MR angiography versus catheter digital subtraction angiography. AJNR Am J Neuroradiol 2008;29:1570-74

17. Roy D, Milot G, Raymond J. Endovascular treatment of unruptured aneurysms. Stroke 2001;32:1998-2004

18. Raymond J, Guilbert F, Weill A, et al. Long-term angiographic recurrences after selective endovascular treatment of aneurysms with detachable coils. Stroke 2003;34:1398-403

19. Dabirzadeh H, Moulton R, Lum C. Long-term durability of coiled cerebral aneurysms in which CTA was used as the sole imaging selection criteria for patients presenting with subarachnoid hemorrhage (SAH). Presented at: Annual Meeting of the Canadian Association of Radiologists, Montreal, Quebec, Canada, April 23-26, 2009

20. Al Azri F, Santos M, Lesiuk H, et al. Contrast-enhanced MR angiography follow-up of intracranial aneurysms treated with "Neuroform" endovascular stent-assisted coiling. Presented at: Annual Meeting of American Society of Neuroradiology, Chicago, Illinois, June 9-14, 2007

21. Broome DR. Nephrogenic systemic fibrosis associated with gadoliniumbased contrast agents: a summary of the medical literature reporting. Eur J Radiol 2008;66:230-34

22. Broome DR, Girgus MS, Baron PW, et al. Gadodiamide-associated nephrogenic systemic fibrosis: why radiologists should be concerned. AJR Am J Roentgenol 2007;188:586-92 